\title{
Biocompatibility Assessments of Surgical sutures: Intracutaneous Reactivity Test in New Zealand White Rabbits
}

\author{
Tangthong Jiraporn ${ }^{1}$, Klinkhamhom Aekkarin', \\ Likitsuntornwong Wanlop ${ }^{2}$ and Amted Nanthana ${ }^{2 *}$
}

${ }^{1}$ Research and Academic support Office, National Laboratory Animal Center, Mahidol University, Thailand, 73170

${ }^{2}$ Veterinary Medical Care Office, National Laboratory Animal Center, Mahidol University, Thailand, 73170

\begin{abstract}
Surgical suture is a medical device used to hold tissues together after an injury or surgery. Application generally involves using a needle with an attached length of thread. The aim of the study was to evaluate local irritant effects to rabbit skin following a single application of test samples of surgical suture. The polar and nonpolar extracts were prepared by using saline solution and olive oil, respectively, after sinking the materials tested $(2.0 \mathrm{~g})$ in $10 \mathrm{ml}$ of the corresponding liquid. Incubation was carried out at the temperature of $37{ }^{\circ} \mathrm{C}$ for $72 \mathrm{~h}$. The saline solution and pure olive oil were used as negative control samples and were incubated under the same conditions as above. Assessments of the extracts from each material were conducted on 3 albino rabbits of the New Zealand breed. On the back of each animal, 5 intracutaneous injections of the extract tested and 5 injections of the control solution, each of $0.2 \mathrm{ml}$, were carried out. The degree of irritation was scored at 4, 24, 48, 72 hours after injection and no skin changes were found. The intracutaneous irritation index (III) was calculated The experimental procedure was conducted according to ISO10993-10
\end{abstract}

Keywords: Surgical suture, Intracutaneous reactivity test.

\section{Introduction}

Polymeric materials have dramatically influenced our day to day life. They find potential in various fields like food packaging, automobiles, water purification etc. ${ }^{[1-3]}$ Application of polymeric biomaterials in medicine has been a thrust area of research owing to the exceptional and superior properties they exhibit. ${ }^{[4]}$ The increased use of polymeric biomaterials in the form of surgical implants, sutures and scaffolds for biomedical applications. ${ }^{[5]}$ 
Surgical suture is a medical device used to hold body tissues together after an injury or surgery. Application generally involves using a needle with an attached length of thread. A number of different shapes, sizes, and thread materials have been developed over its millennia of history. Surgeons, physicians, dentists, podiatrists, eye doctors, registered nurses and other trained nursing personnel, medics, and clinical pharmacists typically engage in suturing. Surgical knots are used to secure the sutures. ${ }^{[6]}$

The primary purpose of suture is to hold apposing tissues together to facilitate and hasten healing process with minimal or no scar formation following an injury or surgical procedure. ${ }^{[7]} \mathrm{A}$ variety of materials such as gold, silver, iron and steel wires, dried animal gut, animal hair (e.g. horse hair), silk, tree bark and plant fiber (e.g. linen, cotton) were used as suture materials in the past, while some of them are still use. The recent has witnessed the use of various synthetic biomaterials such as polydioxanone, poly(lactic-co-glycolic acid) as suture materials. ${ }^{[8]}$

To minimize any potential hazards to the patients, it is essential that biocompatibility assessments be conducted for surgical suture made from Polyglycolide-Co-L-Lactide that are used in medical devices. The common tests are used to measure biocompatibility: ISO10993-10, Biological Evaluation of Medical Devices $(2010){ }^{[9]}$

\section{Animals and Husbandry}

Irritation and intracutaneous tests maybe applicable where determination of irritation by dermal or mucosal irritation tests is appropriate. Albino rabbits are most commonly used. Because intracutaneous reactivity test focus on determining the biological response of leachable agents that may be present in biomaterials.

Animals and husbandry were conducted based on the test guidance of The International Organization for Standardization 10993-2, Biological Evaluation of Medical Devices-Part 2: Animal Welfare Requirement, $2006^{[10]}$, The International Organization for Standardization 10993-10, Biological evaluation of medical devices Part 10 Test for irritation and delayed type hypersensitivity, 2010 ${ }^{[9]}$ and Guidelines of "Guide for the care and use of laboratory animals" (Institute of laboratory animal resources, National academic press 2011; NIH publication number \#85-23, revised 2011).

Three healthy young female New Zealand White rabbits of body weight in range 2,030-2,242 g were obtained from Office of Laboratory Animal Production, NLAC, Mahidol University, Thailand. The animals were kept under standard conditions 12:12 (light : dark cycles) at 22 $\pm 30 \mathrm{C}$ and 30-70\% relative humidity. The animals were housed individually in stainless cages. The animals were fed with feed and chlorinated water $a d$ libitum. All the animals were acclimatized for 5 days prior to the study. The study was approved by National Laboratory Animal Center Animal Care and Use Committee (NLAC-ACUC), Mahidol University; Thailand.

\section{Preparation of the test material extracts}

The surgical suture (Polyglycolide-Co-L-Lactide) and control item preparation was conducted based on the test guidance of the International Organization for Standardization 10993-12, Biological Evaluation of Medical Devices - Part 12: Sample Preparation and Reference Materials, 2007. ${ }^{[1]}$

Polar solvent (Physiological saline) and Non polar solvent (olive oil) were used as a control item.

Two grams of the surgical suture (Polyglycolide-Co-L-Lactide) was extracted in Polar solvent and Non polar solvent. The solution were performed in a water bath at $37^{\circ} \mathrm{C}$ for 72 hours. Polar solvent and Non polar solvent which had no contact with the surgical suture were use as negative control and were incubated under the same conditions as above. The extracts were used within 4 hours to perform the test procedure. ${ }^{[11]}$

\section{Intracutaneous reactivity test}

Fur on the back of each animal was clipped with an electric clipper $16-24$ hours prior to exposure (about $10 \times 15 \mathrm{~cm}$ ). On the day of exposure, the clipped area was separated to 4 sites on each animal. The 
surgical suture extraction and control item were intracutaneous (Intradermal) applied to the test sites and control sites respectively.

An approximate of $0.2 \mathrm{ml}$ of surgical suture extraction was extracted in Physiological saline and olive oil were aseptically injected intracutaneously into five sites on upper left hand side and lower left hand side, respectively. The control item (Physiological saline and olive oil) were aseptically injected intracutaneously at a dose of $0.2 \mathrm{ml}$ into five sites on the upper right hand side and lower right hand side, respectively. Skin reaction (erythema and oedema), at the site of application was subjectively assessed and scored at 4, 24, 48 and 72 hours after intracutaneous injection of the test item. During the observation period, the animals were handled with care to advoid touching the injection sites. The reaction at the site of injections were assessed and scored according to the following numerical sytem. (Table 1.) ${ }^{[9]}$

\section{Evaluation of result}

After the $72 \mathrm{~h}$ grading, all erythema grades plus oedema grades $24(+2) \mathrm{h}, 48(+2) \mathrm{h}$ and $72(+2)$ are totalled separately for each surgical suture extraction and control item for each animal. To calculate the score of a surgical suture extraction or control item on each individual animal, divide each of the totals by 15 (3 scoring time point $\mathrm{x} 5$ test item or control item injection sites). To determine the overall mean score for each surgical suture extraction and each corresponding control item, add the scores for the three animals and divide by three. The final test item score can be obtained by subtracting the score of the control item from the test item score. The requirements of the test item are met if the final test item score is 1.0 or less. If at any observation period the average reaction to the surgical suture extraction is questionably greater than the average reaction to the control item.

\section{Table 1. Scoring system for skin reaction}

\begin{tabular}{ll}
\hline Reaction & Irritation score \\
\hline Erythema and eschar formation & \\
\hline No erythema & 0 \\
\hline Very slight erythema (barely perceptible) & 1 \\
\hline Well defined erythema & 2 \\
\hline Moderate erythema & 3 \\
\hline Severe erythema & 4 \\
\hline Oedema formation & 0 \\
\hline No oedema & 1 \\
\hline Very slight oedema (barely perceptible) & 2 \\
\hline Well defined oedema & 3 \\
\hline Moderate oedema & 4 \\
\hline Severe oedema & 8 \\
\hline Maximum possible score for irritation & \\
\hline Other adverse changes at the skin sites shall be recorded and reported & \\
\hline
\end{tabular}

Table 2. Primary or cumulative irritation index categories in a rabbits

\begin{tabular}{cc}
\hline Mean score & Response category \\
\hline 0 to 0.4 & Negligible \\
\hline 0.5 to 1.9 & Slight \\
\hline 2.0 to 4.9 & Moderate \\
\hline 5.0 to 8.0 & Severe \\
\hline
\end{tabular}




\section{Result}

All animals appeared active and healthy during the study. Apart from the dermal irritation noted below, there were no abnormal behavior. No edema was observed at any treated dose site. Tables 3. -8 . show the irritation potential of the physiological saline and olive oil extracts of suture. It was found that the animals did not show any grade of erythema and/or edema after intradermal injection of the material extracts studied. The average irritation score induced by the physiological saline and olive oil extract of surgical sutures was 0.0. The irritation potential induced by either material extracts was comparable to controls.

Macroscopic finding: Skin erythema and edema were observed in area was administrated of olive oil (non polar) extract $(0.2 \mathrm{ml})$ of suture and area was administrated of olive oil (non polar) except area was administrated of Physiological saline (polar) extract $(0.2 \mathrm{ml})$ of suture and area was administrated of Physiological saline (polar). Skin erythema was small lesion surround injecting needle size. Edema was skin elevation by yellow solution under each injection size. Skin operation, there were mild to moderate petechial hemorrhage.

Table 3. Irritation effects of intracutaneous (i.c.) administration of olive oil (non polar) extract (0.2 ml) of surgical suture (Polyglycolide-Co-L-Lactide) in rabbit No. 1 compared with control.

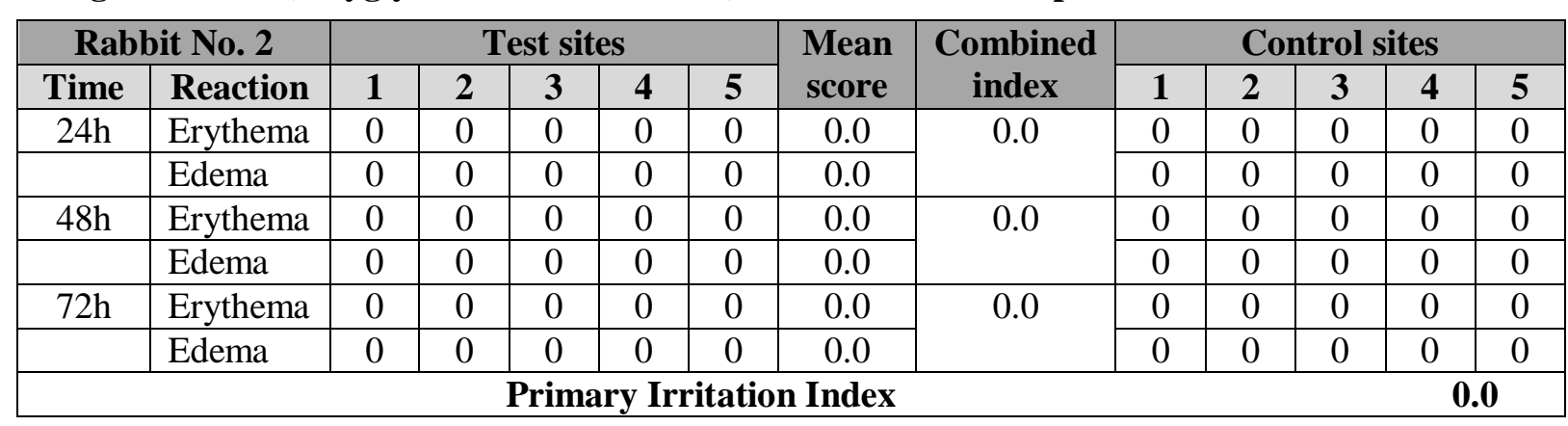

Table 4. Irritation effects of intracutaneous (i.c.) administration of olive oil (non polar) extract (0.2 ml) of surgical suture (Polyglycolide-Co-L-Lactide) in rabbit No. 2 compared with control.

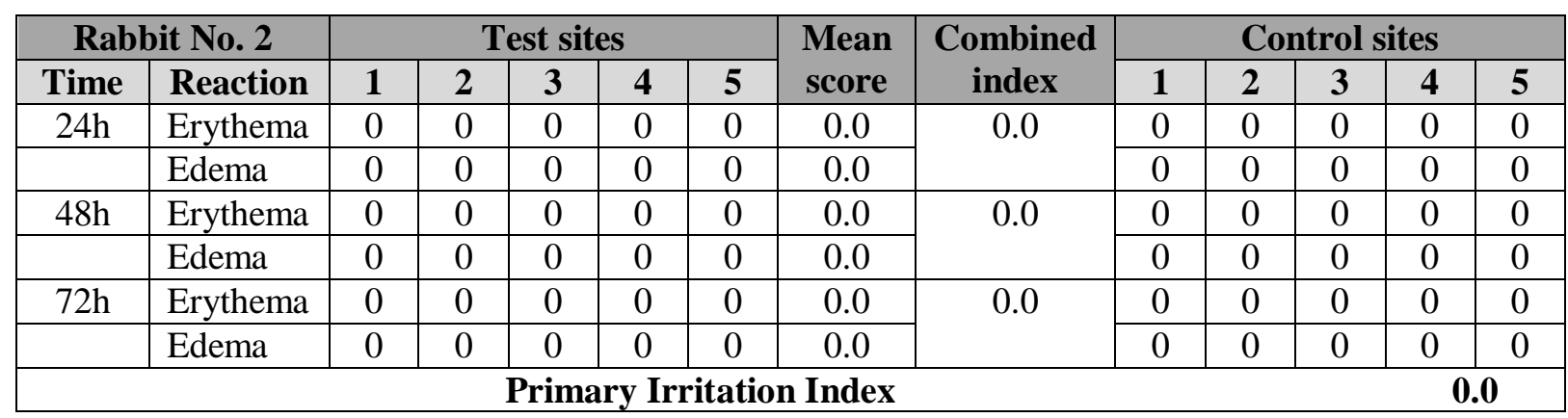

Table 5. Irritation effects of intracutaneous (i.c.) administration of olive oil (non polar) extract (0.2 ml) of surgical suture (Polyglycolide-Co-L-Lactide) in rabbit No. 3 compared with control.

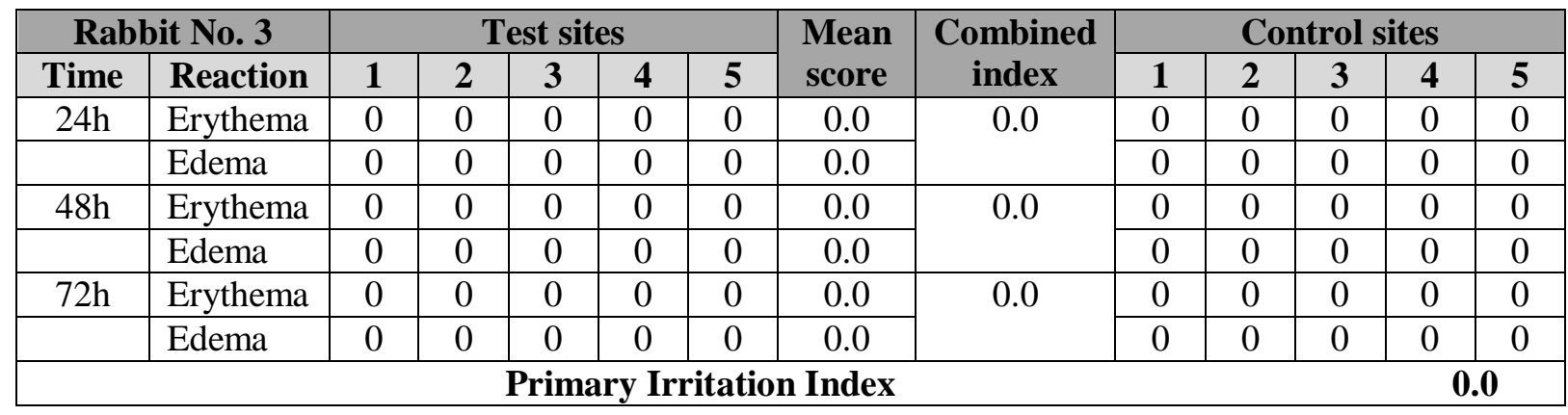


Table 6. Irritation effects of intracutaneous (i.c.) administration of Physiological saline (polar) extract $(0.2 \mathrm{ml})$ of surgical suture (Polyglycolide-Co-L-Lactide) in rabbit No. 1 compared with control.

\begin{tabular}{|c|c|c|c|c|c|c|c|c|c|c|c|c|c|}
\hline \multicolumn{2}{|c|}{ Rabbit No. 1} & \multicolumn{5}{|c|}{ Test sites } & \multirow{2}{*}{$\begin{array}{l}\text { Mean } \\
\text { score }\end{array}$} & \multirow{2}{*}{$\begin{array}{l}\text { Combined } \\
\text { index }\end{array}$} & \multicolumn{5}{|c|}{ Control sites } \\
\hline Time & Reaction & 1 & 2 & 3 & 4 & 5 & & & 1 & 2 & 3 & 4 & 5 \\
\hline \multirow[t]{2}{*}{$24 \mathrm{~h}$} & Erythema & 0 & 0 & 0 & 0 & 0 & 0.0 & \multirow[t]{2}{*}{0.0} & 0 & 0 & 0 & 0 & 0 \\
\hline & Edema & 0 & 0 & 0 & 0 & 0 & 0.0 & & 0 & 0 & 0 & 0 & 0 \\
\hline \multirow[t]{2}{*}{$48 \mathrm{~h}$} & Erythema & 0 & 0 & 0 & 0 & 0 & 0.0 & \multirow[t]{2}{*}{0.0} & 0 & 0 & 0 & 0 & 0 \\
\hline & Edema & 0 & 0 & 0 & 0 & 0 & 0.0 & & 0 & 0 & 0 & 0 & 0 \\
\hline \multirow[t]{2}{*}{$72 \mathrm{~h}$} & Erythema & 0 & 0 & 0 & 0 & 0 & 0.0 & \multirow[t]{2}{*}{0.0} & 0 & 0 & 0 & 0 & 0 \\
\hline & Edema & 0 & 0 & 0 & 0 & 0 & 0.0 & & 0 & 0 & 0 & 0 & 0 \\
\hline
\end{tabular}

Table 7. Irritation effects of intracutaneous (i.c.) administration of Physiological saline (polar) extract $(0.2 \mathrm{ml})$ of surgical suture (Polyglycolide-Co-L-Lactide)in rabbit No. 2 compared with control.

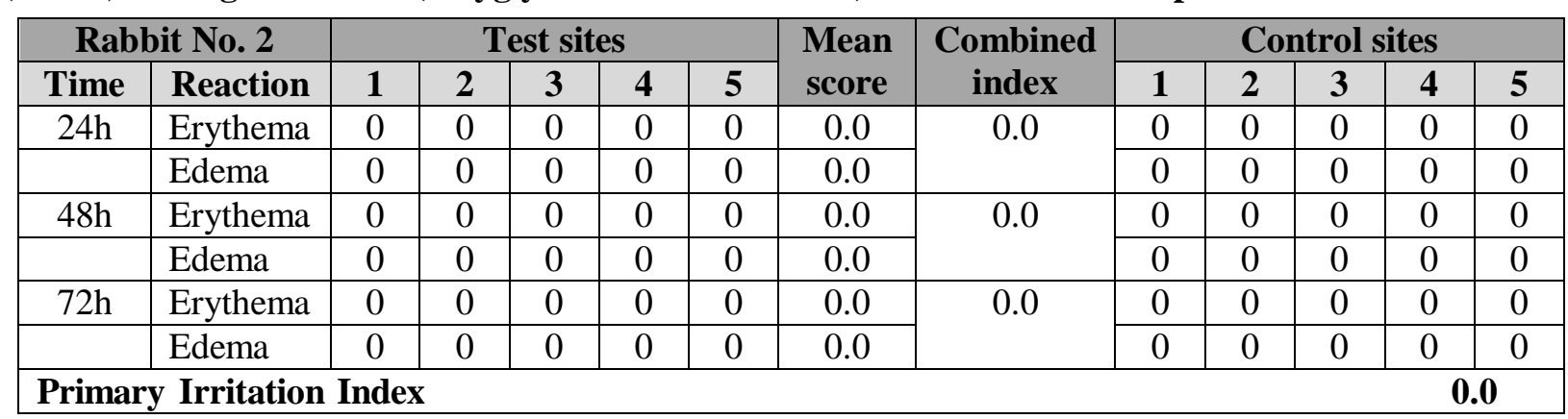

Table 8. Irritation effects of intracutaneous (i.c.) administration of Physiological saline (polar) extract $(0.2 \mathrm{ml})$ of surgical suture (Polyglycolide-Co-L-Lactide)in rabbit No. 3 compared with control.

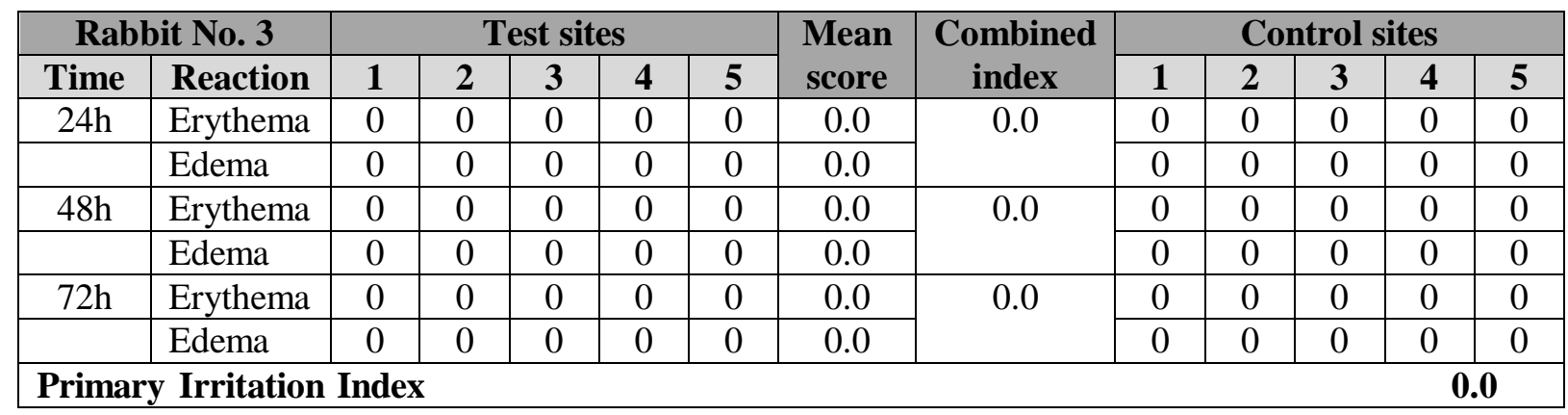

\section{Conclusion and Discussion}

Biocompatibility is a general term used to describe the suitability of a material for exposure to the body or bodily fluids. Biocompatibility testing is essential for all materials that will be used in medical devices to minimize any potential hazards to the patient. A material will be considered biocompatible if it allows the body to function without any complications, such as allergic reactions, irritation or other adverse side effects. The present study can by considered as a part of the whole biocompatibility testing.

Surgical sutures as a medical device in wound management and recent advancements have expanded its applicability and efficacy. Major progress in this front can be attributed toward the technological advancements in materials science. Polymers hold a significant potential with their high flexibility giving rise to diverse suture materials with excellent physical and mechanical properties. In addition, to better handling qualities and desired modifications, it should also be non carcinogenic, nontoxic, free of allergens, and importantly it should not evoke any adverse response in the host tissues. To meet these requirements, it is necessary to conduct detailed pre-clinical studies and evaluate the safety and efficacy in human trials on these 
emerging sutures. The next generation of suture materials, an outcome of multidisciplinary efforts has immense potential to impact surgical outcomes and wound management.

\section{Acknowledgements}

This study was supported by Thailand Center of Excellence for Life Sciences and National Laboratory Animal Center, Mahidol University, Thailand.

\section{References}

1. Baldino L Cardea S Reverchon E. Biodegradable membranes loaded with curcumin to be used as engineered independent devices in active packaging. Journal of the Taiwan Institute of Chemical Engineers. 2017; 71: 518-526doi:10.1016/j.jtice.2016.12.020.

2. Soyekwo F Zhang Q Gao R, Qu Y Lin C et al. Cellulose nanofiber intermediary to fabricate highlypermeable ultrathin nanofiltration membranes for fast water purification. The Journal of Membrane Science. 2017; 524: 174-185, doi:10.1016/j.memsci.2016.11.019..

3. Evangelisti S Tagliaferri C Brett DJ Lettieri P. Life cycle assessment of a polymer electrolyte membrane fuel cell system for passenger vehicles. The Journal of Cleaner Production. 2017; 142(4): 4339-4355, doi:10.1016/j.jclepro.2016.11.159.

4. Teo AJ Mishra A Park I Kim YJ Park WT Yoon YJ. Polymeric biomaterials for medical implants and devices. ACS Biomaterials Science \& Engineering. 2016; 2(4): 454-472, doi:10.1021/acsbiomaterials.5b00429.

5. Wong JY Bronzino JD. Peterson DR. Biomaterials: Principles and Practices. CRC Press. 2012.

6. Rumyana $S$ and Nikolai D. Assessment of surgical sutures Polymed ${ }^{\circledR}$ by intracutaneous irritation test in rabbits. Interdisciplinary Toxicology. 2013; 6(2): 99-102, doi:10.2478/intox-2013-0017.

7. Greenberg JA Clark RM. Advances in suture material for obstetric and gynecologic surgery. Reviews in Obstetrics \& Gynecology. 2009; 2(3): 146-158.

8. International Organization for Standardization. Biological evaluation of medical devices Part 10 Test for irritation and delayed type hypersensitivity. 2010

9. International Organization for Standardization 10993-2. Biological Evaluation of Medical DevicesPart 2: Animal Welfare Requirement. 2006

10. International Organization for Standardization. Biological evaluation of medical devices Part 12 Sample preparation and reference material: Clause 10. Preparation of extracts of sample. 2010. 